\title{
HMMR is differentially expressed in lymph node metastasis in human breast cancer.
}

Shahan Mamoor, MS ${ }^{1}$

${ }^{1}$ shahanmamoor@gmail.com

East Islip, NY USA

Metastasis to the brain is a clinical problem in patients with breast cancer ${ }^{1-3}$. Between the breast and the brain reside the secondary lymphoid organ, the lymph nodes. We mined published microarray data ${ }^{4,5}$ to compare primary and metastatic tumor transcriptomes for the discovery of genes associated with metastasis to the lymph nodes in humans with metastatic breast cancer. We found that the hyaluronan mediated motility receptor, HMMR, was among the genes whose expression was most different in the lymph node metastases of patients with metastatic breast cancer as compared to primary tumors of the breast. HMMR mRNA was present at increased quantities in lymph node metastases as compared to primary tumors of the breast. Importantly, expression of HMMR in primary tumors of the breast was correlated with patient distant metastasis-free survival. Modulation of HMMR expression may be relevant to the biology by which tumor cells metastasize from the breast to the lymph nodes and the brain in humans with metastatic breast cancer.

Keywords: breast cancer, metastasis, brain metastasis, central nervous system metastasis, lymph node metastasis, hyaluronan mediated motility receptor, HMMR, systems biology of breast cancer, targeted therapeutics in breast cancer. 
One report described a 34\% incidence of central nervous system metastases in patients treated with trastuzumab for breast cancer ${ }^{2}$. More recently, the NEfERT-T clinical trial ${ }^{6}$ which compared administration of either neratinib or trastuzumab in conjunction with paclitaxel demonstrated that in a randomized, controlled setting, in breast cancer patients treated with neratinib, not only was the incidence of central nervous system recurrence significantly lower, the time to central nervous system metastasis was significantly delayed as compared to patients administered trastuzumab ${ }^{6}$. The alarmingly high rate of central nervous system metastasis described, as well as data, both anecdotal ${ }^{2}$ and from a randomized, controlled setting ${ }^{6}$ illustrating that treatment with trastuzumab may be associated with these events demands an enhanced understanding of the transcriptional makeup of brain and lymph node metastatic tissues to support identification of therapeutic targets, whether they are treatment related or not. The lymph nodes reside between the breast and the brain. We performed a global comparative analysis of lymph node metastatic tumor tissues in patients with metastatic breast cancer, compared to primary tumors and normal breast tissues ${ }^{4,5}$. We discovered transcriptome-wide differential expression of the gene encoding hyaluronan mediated motility receptor, HMMR, in lymph node metastatic tissues of patients with metastatic breast cancer.

\section{$\underline{\text { Methods }}$}

We used datasets GSE10893 ${ }^{4}$ and GSE1 $24648^{5}$ for this global differential gene expression analysis of brain metastatic breast cancer in conjunction with GEO2R. GSE10893 was generated using Agilent-011521 Human 1A Microarray G4110A technology with $n=71$ primary breast tumors and $n=7$ lymph node metastases from patients with breast cancer; analysis was performed using platform GPL887. GSE124648 was generated using Affymetrix Human Genome U133A array technology with $n=10$ normal breast tissues and $n=44$ lymph node metastases from patients with breast cancer; analysis was performed using platform GPL96. The Benjamini and Hochberg method of $p$-value adjustment was used for ranking of differential expression but raw $p$-values were used to assess statistical significance of global differential expression. Log-transformation of data was auto-detected, and the NCBI generated category of platform annotation was used. A statistical test was performed to evaluate whether HMMR gene expression was significantly different between primary tumors of the breast and lymph node metastases in humans with breast cancer using a two-tailed t-test. For Kaplan-Meier survival analysis, we used the Kaplan-Meier plotter online tool ${ }^{7}$ for correlation of HMMR mRNA expression levels with distant metastasis-free survival in $n=2765$ patients with breast cancer.

\section{$\underline{\text { Results }}$}

We performed global comparative transcriptome analysis of metastatic tumor tissues of patients with metastatic breast cancer using published microarray data ${ }^{4,5}$ to describe the transcriptional landscape of metastasis in human breast cancer in an unbiased fashion and for the discovery of novel therapeutic targets.

\section{HMMR is differentially expressed in the lymph node metastases of patients with metastatic breast} cancer.

Through blind, systems-level analysis of published microarray data ${ }^{4}$, we identified the hyaluronan mediated motility receptor, encoded by HMMR, as a differentially expressed gene in the lymph node metastatic tissues of humans with breast cancer (Table 1). When sorting each of the genes expressed in lymph node metastases based on significance of difference as compared to primary tumors of the breast in patients with breast cancer, HMMR ranked 222 out of 18034 total transcripts (Chart 1), equating to $98.8 \%$ differential expression. Differential expression of HMMR in the lymph node metastases of patients with metastatic breast cancer was statistically significant (Chart $1 ; p=2.72 \mathrm{e}-03$ ). 
We queried a second microarray dataset ${ }^{5}$ to attempt to validate transcriptome-wide differential expression of HMMR in the lymph node metastases of patients with metastatic breast cancer. We again observed HMMR to be among the genes expressed most quantitatively differently in the lymph node metastases of patients with metastatic breast cancer as compared to primary tumors of the breast. When sorting each of the genes expressed in lymph node metastases based on significance of difference as compared to normal breast tissues, HMMR ranked 4386 out of 22283 total transcripts (Chart 2), equating to $80.3 \%$ differential expression. Differential expression of HMMR in the lymph node metastases of patients with metastatic breast cancer was statistically significant (Chart $2 ; p=3.71 \mathrm{e}-03$ ).

\section{HMMR is expressed at higher levels in the lymph node metastases of patients with metastatic breast} cancer.

\section{HMMR expression is correlated with survival outcomes in human breast cancer.}

We performed Kaplan-Meier survival analysis ${ }^{7}$ in $n=2765$ breast cancer patients, to evaluate whether HMMR tumor expression was correlated with survival outcomes in human breast cancer. We observed a significant correlation between primary tumor expression of HMMR and distant metastasis-free survival (DMFS) in human breast cancer (Figure 2). Patients whose primary tumors expressed low levels of HMMR possessed median DMFS of 125.65 months, while patients whose tumors expressed high levels of HMMR possessed median DMFS of 51.88 months. This difference in DMFS based on HMMR tumor expression in human breast cancer was statistically significant (Figure 2, Chart 3; logrank $p$-value: 6.6e-08 (hazard ratio: 1.53 (1.31-1.79))).

Thus, by mining published microarray data ${ }^{4,5}$ in an unbiased and systematic fashion, we identified HMMR as among the genes whose expression was most different, transcriptome-wide, in the lymph node metastases of patients with breast cancer, when compared to primary tumors; we observed significantly increased expression of HMMR in lymph node metastases as compared to primary tumors of the breast. Further, we found a correlation between HMMR expression and patient distant metastasis-free survival in human breast cancer.

\section{$\underline{\text { Discussion }}$}

We provided evidence here that hyaluronan mediated motility receptor, encoded by HMMR, is among the genes whose expression is most different in the lymph node metastases of patients with metastatic breast cancer as compared to primary tumors of the breast, that HMMR mRNA is present at significantly increased quantities in lymph node metastatic tissues as compared to primary tumors of the breast, and that primary tumor HMMR expression is correlated with patient survival outcomes in human breast cancer. Evaluation of the effects of genetic depletion of HMMR in mouse models of metastatic breast cancer on metastasis to the lymph nodes and to the central nervous system is merited. Modulation of HMMR expression may be relevant to the processes by which breast cancer cells exit the breast, enter 


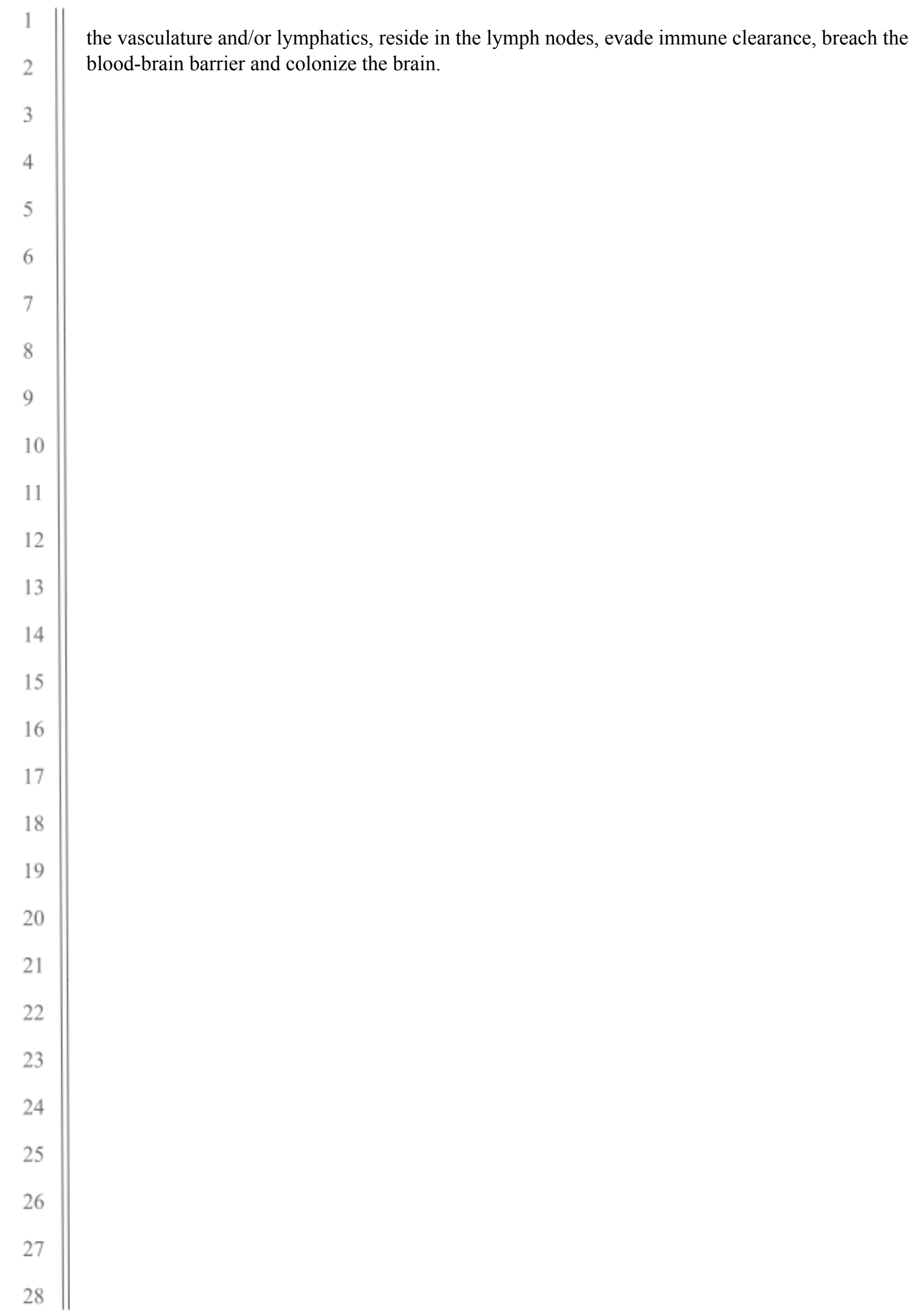




\section{$\underline{\text { References }}$}

1. Lin, N.U., Amiri-Kordestani, L., Palmieri, D., Liewehr, D.J. and Steeg, P.S., 2013. CNS metastases in breast cancer: old challenge, new frontiers.

2. Bendell, J.C., Domchek, S.M., Burstein, H.J., Harris, L., Younger, J., Kuter, I., Bunnell, C., Rue, M., Gelman, R. and Winer, E., 2003. Central nervous system metastases in women who receive trastuzumab-based therapy for metastatic breast carcinoma. Cancer, 97(12), pp.2972-2977.

3. Tsukada, Y., Fouad, A., Pickren, J.W. and Lane, W.W., 1983. Central nervous system metastasis from breast carcinoma autopsy study. Cancer, 52(12), pp.2349-2354.

4. Weigman, V.J., Chao, H.H., Shabalin, A.A., He, X., Parker, J.S., Nordgard, S.H., Grushko, T., Huo, D., Nwachukwu, C., Nobel, A. and Kristensen, V.N., 2012. Basal-like Breast cancer DNA copy number cancer research and treatment, 133(3), pp.865-880.

5. Sinn, B.V., Fu, C., Lau, R., Litton, J., Tsai, T.H., Murthy, R., Tam, A., Andreopoulou, E., Gong, Y., Murthy, R. and Gould, R., 2019. SET ER/PR: a robust 18-gene predictor for sensitivity to endocrine therapy for metastatic breast cancer. NPJ breast cancer, 5(1), pp.1-8.

6. Awada, A., Colomer, R., Inoue, K., Bondarenko, I., Badwe, R.A., Demetriou, G., Lee, S.C., Mehta, A.O., Kim, S.B., Bachelot, T. and Goswami, C., 2016. Neratinib plus paclitaxel vs trastuzumab plus paclitaxel in previously untreated metastatic ERBB2-positive breast cancer: the NEfERT-T randomized clinical trial. JAMA oncology, 2(12), pp.1557-1564.

7. Györffy, B., Lanczky, A., Eklund, A.C., Denkert, C., Budczies, J., Li, Q. and Szallasi, Z., 2010. An online survival analysis tool to rapidly assess the effect of 22,277 genes on breast cancer prognosis using microarray data of 1,809 patients. Breast cancer research and treatment, 123(3), pp.725-731. 
Rank: 222

Probe ID: 12527

p-value: $2.72 \mathrm{e}-03$

t: -3.0948312

B: -1.6553

Gene: HMMR

Gene name: hyaluronan mediated motility receptor

Chart 1: HMMR is differentially expressed in lymph node metastatic breast cancer when comparing lymph node metastases to primary tumors of the breast.

The rank of global differential expression, the probe/transcript ID, the $p$-value with respect to differential expression transcriptome-wide, $\mathrm{t}$, a moderated $\mathrm{t}$-statistic, $\mathrm{B}$, the log-odds of differential expression between the groups compared, the gene and gene name are listed in this chart.

Rank: 4386

Probe ID: 207165_at

p-value: $3.71 \mathrm{e}-03$

t: -3.029814

B: -2.685156

Gene: HMMR

Gene name: hyaluronan mediated motility receptor

Chart 2: HMMR is differentially expressed in lymph node metastatic breast cancer when comparing lymph node metastases to normal breast tissues.

The rank of global differential expression, the probe/transcript ID, the $p$-value with respect to differential expression transcriptome-wide, $\mathrm{t}$, a moderated t-statistic, $\mathrm{B}$, the log-odds of differential expression between the groups compared, the gene and gene name are listed in this chart. 







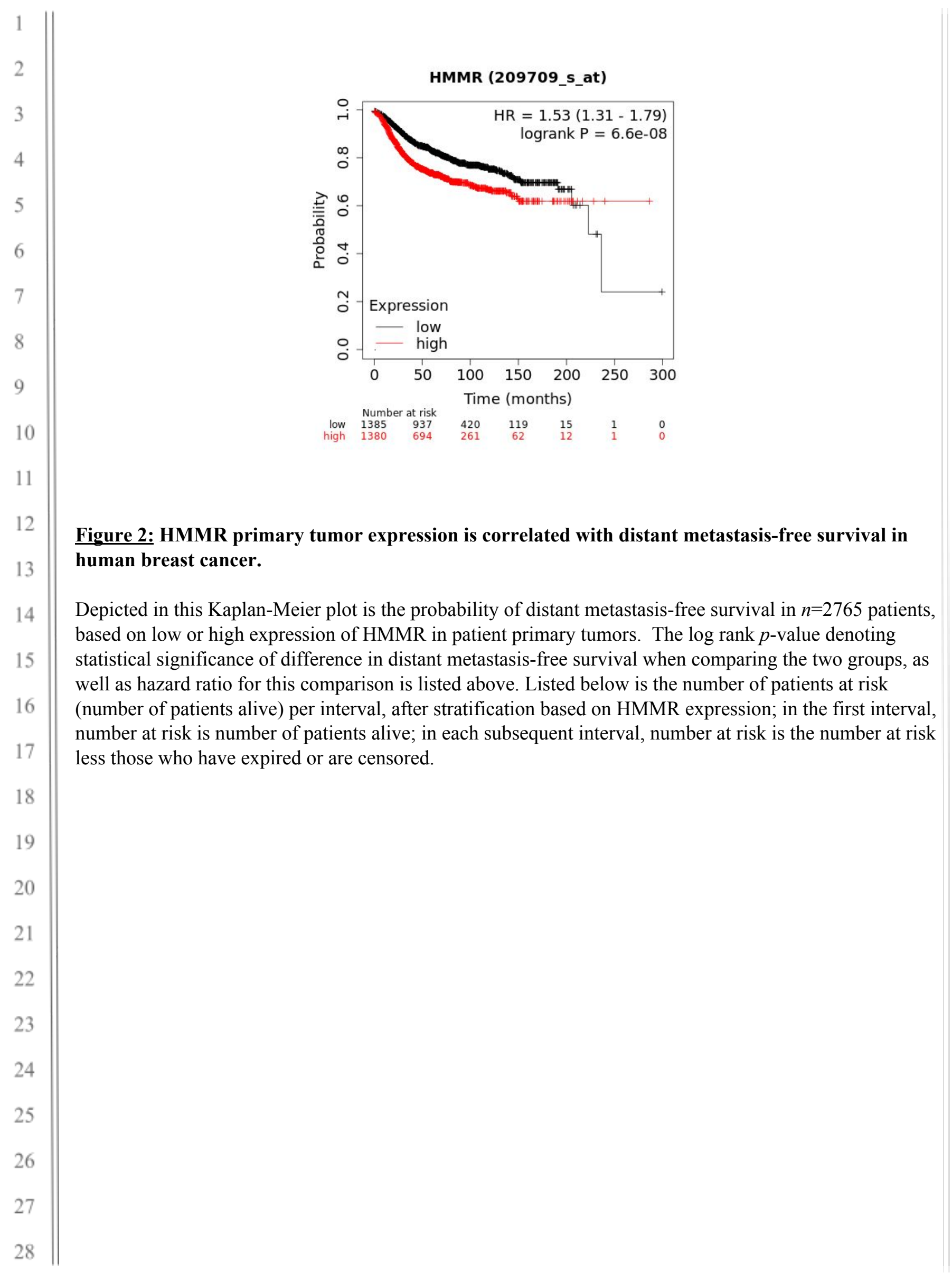




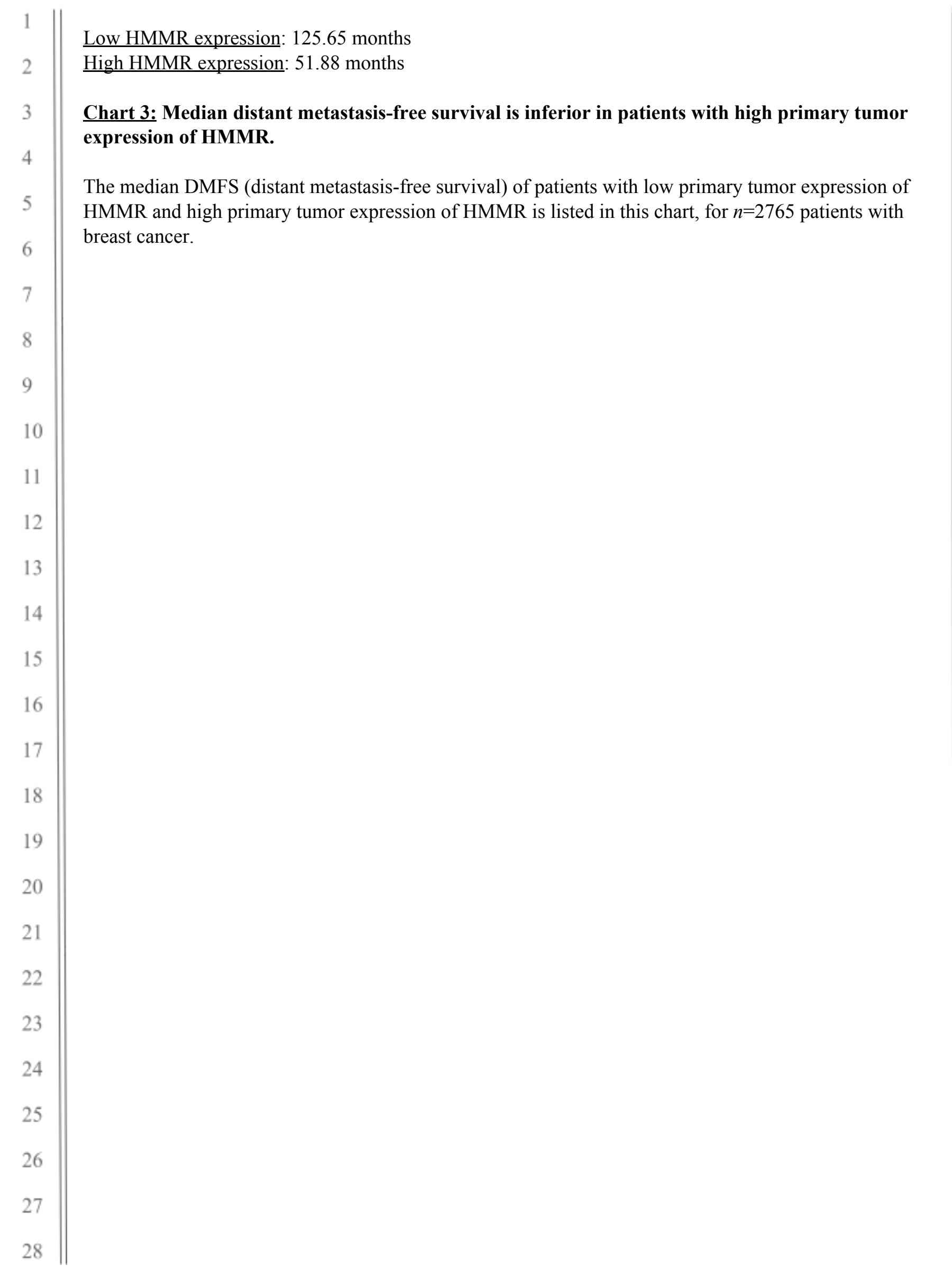

